\title{
Topical delivery of mesenchymal stem cells and their function in wounds
}

\author{
J Michael Sorrell and Arnold I Caplan*
}

\begin{abstract}
Mesenchymal stem cells are a heterogeneous population of fibroblast-like cells found in most adult organs. However, most of our current knowledge is based on cells of bone marrow or interstitial adipose tissues. These cells are capable of differentiation along various mesenchymal lineages. In addition, they have demonstrated therapeutic characteristics in wounds and ischemic situations. The therapeutic characteristics of these cells are activated upon their entering wounds or other damaged tissues. A current problem is the development of strategies that ensure that these cells reach wound beds in a timely fashion and in sufficient numbers to maximize their therapeutic benefits. Currently, there are two basic delivery methods: systemic infusion of cells into the vascular circulation and direct application of therapeutic cells to wound sites. Skin wounds are optimal candidates for the topical delivery approach. However, the methods by which therapeutic cells are delivered to such wounds vary. This review outlines the basic methods used to deliver therapeutic cells to skin and other wounds. Upon entering wounds, therapeutic cells interact with other wound cells through paracrine mechanisms that are not yet well understood. Nonetheless, interactions with vascular endothelial cells and immunomodulation appear to play significant roles in accelerating wound healing and in reducing scar formation upon the completion of the healing process. Although the phenomenological body of evidence indicating the efficacy of therapeutic cells is substantial, considerable work is still required to better determine the molecular and cellular functions of these cells and to assess their fate and the long-term consequences of their application.
\end{abstract}

*Correspondence: aic@case.edu

Department of Biology, Skeletal Research Center, Case Western Reserve University, 2080 Adelbert Road, Cleveland, OH 44106, USA

\section{Introduction: what are mesenchymal stem cells?}

The term mesenchymal stem cell (MSC) applies to adult fibroblast-like cells that differentiate along multiple mesenchymal pathways when exposed to proper stimuli $[1,2]$. These cells were identified first in murine bone marrow as plastic-adherent cells that formed fibroblast colony-forming units [3]. Other investigators began to adapt similar adherent populations as feeder layers for the propagation of various hematopoietic cell lineages [4]. This usage provided the first glimpse of the ability of MSCs to secrete potent bioactive factors that enabled them to regulate the function of other types of cells. This cellular regulatory capacity underlies the current notion that MSCs possess therapeutic potential to promote the healing of wounds and ischemic tissues [1,2]. This implies that MSCs can also function as therapeutic cells that modulate microenvironments and immunological competence, accelerate wound repair, and reduce fibrosis or scar formation or both. A number of recent studies have been translating this concept into experimental studies and further into clinical applications. To date, such applications include cardiovascular disease and myocardial infarction; brain and spinal cord injury; cartilage, bone, and tendon repair; Crohn disease; and skin wound repair [5-7].

The phenotypic definition of MSCs has been hampered by the heterogeneity of this population [8,9]. Heterogeneity occurs among cells harvested from a single anatomic site and also occurs between cells harvested from different anatomic sites. Bone marrow and adipose tissues are currently the major sources for MSCs that are being used for preclinical and clinical studies. Adipose stromal cells (ASCs), though exhibiting differences, still share basic characteristics with bone marrow-derived cells [10-12]. As with MSCs, ASCs have been employed in animal wound repair models and in preliminary clinical studies such as myocardial infarction, Crohn disease, and skin wound repair [13]. Cells with MSC characteristics have also been identified in multiple adult organs, where these cells are associated with vasculature. These cells include both pericytes in the microvasculature and adventitial fibroblast-like cells that surround the larger blood vessels [13-15]. 
Minimal criteria for defining MSCs and ASCs are that they adhere to tissue culture plastic, express cell surface markers for CD105, CD73, and CD90, and fail to express cell surface markers for CD45, CD34, CD14, CD11b, CD79a, and CD19 $[12,16,17]$. In addition, these cells are negative for class II major histocompatibility markers [2,5]. The evolution of therapeutic approaches using MSCs has raised new issues regarding the identification of these cells. The abilities to produce paracrine factors and to interact with other types of cells typically found in wounds require a more extensive investigation to develop a functional profile for these cells.

\section{Delivery of exogenous mesenchymal stem cells to wound/ischemic sites}

Most therapeutic applications of MSCs to wound/ ischemic targets dictate that exogenous (for example, culture-expanded) populations be delivered using either systemic or direct/topical approaches [2,18]. Systemic delivery mimics the route of endogenous MSCs via the circulatory system with final homing to target sites. During vascular transit, MSCs risk being taken out of circulation, on either a temporary or a permanent basis, in organs such as the lungs, spleen, and liver [5]. This may either delay their transit or reduce the numbers of cells that finally appear at target sites. Upon reaching their target site (or sites), MSCs must exit the vasculature to enter the connective tissue stromal region where their principal functions occur [5].

An alternate method for the delivery MSCs to wound/ ischemic sites is through direct or topical delivery [19]. This method is fundamentally different from systemic delivery in that applied MSCs either migrate into the wound bed via non-vascular routes or release bioactive factors from a bandage or other type of carrier at the surface of the wound. A limitation of direct/topical delivery is the accessibility of the target site. Skin is an example of a highly accessible target site in which large surface area wounds and chronic non-healing wounds are amenable to topical MSC therapy $[19,20]$. Topical administration of MSCs is generally inapplicable for internal organs. However, direct injection of concentrated cells has been used to deliver cells to internal organs [9]. Nonetheless, this is an invasive procedure with attendant risks.

\section{Direct/topical delivery of exogenous mesenchymal stem cells}

For direct/topical delivery to succeed, a highly concentrated population of cells must be either placed onto the surface of the wound or injected immediately adjacent to the wound. The timing of this administration may also be important in that applied MSCs must functionally interact with wound cells at critical stages of the healing process. Exactly what these interactions are and when they occur are currently areas of research interest. The importance of delivering a critical number of cells has been described by Falanga and colleagues [19]. The mechanisms by which MSCs are concentrated at wound sites vary among studies. Hanson and colleagues [20] have summarized delivery methods that have been employed in small-scale clinical studies. These methods include injection into the wound, inclusion in a topical fibrin spray, and incorporation in a collagen sponge $[19,21,22]$. Similar methods have been employed in preclinical studies $[19,23,24]$. These studies all report improvement in wound healing. However, the variations in delivery methods make it difficult to critically assess cellular and molecular function of MSCs at wound sites. The optimal cellular delivery method for a preclinical or clinical study will likely depend on the type and location of the wound.

Because of its exposed nature, skin has been a target for direct/topical delivery in both preclinical and clinical studies. Minor skin wounds heal well without treatment; however, extensive full-thickness wounds such as those that result from polytrauma or burns require intervention to heal in a timely manner without excessive scarring [25]. Autologous grafts have been used in such situations, but there is a limit to the amount of skin available for grafting. Autologous and allogeneic skin substitutes have also been employed [26]. The integration of MSCs into skin substitutes may provide a novel approach in accelerating the healing of these wounds [27]. Chronic nonhealing skin wounds have a deficit in vascularity and support for vascular function $[19,20]$. Use of cell-based treatments for these wounds is increasing. The inclusion of MSCs into the cellular treatment models for chronic wounds has demonstrated promising results $[19,20]$. Cellular therapeutics has multiple aims: to hasten repair, to dampen overexuberant inflammatory events, and to reduce or eliminate scar formation; all of these aims require a multifunctional cellular population. In some situations, such as for chronic non-healing wounds, the objective of cellular therapy is to reverse those cellular and vascular events that compromise repair $[6,19]$. These cells are typically administered in a direct/topical manner. As with other cellular-based therapies, the cellular and molecular mechanisms by which MSCs exert their influences are still under investigation.

\section{Multiple approaches for direct/topical delivery}

A critical issue for the direct/topical delivery of therapeutic cells is the mechanism by which these cells become integrated into the wound. Three examples for the delivery of these cells to preclinical skin wounds are provided. In each example, therapeutic cells become integrated into the wound tissue. 
Stoff and colleagues [23] injected concentrated human MSCs (hMSCs) into sites immediately adjacent to incisional wounds made in the skin of rabbits. These hMSCs migrated from the injection sites and traversed the dermal-epidermal junction region of the wound by day 14 and had reached the junction between the margin of the wound bed and the underlying fascia by day 21 . These results provide evidence that MSCs were capable of migration through connective tissues and further suggested that specific attractive mechanisms were at play. The wounds treated with hMSCs regained $52 \%$ of the normal tensile strength of skin compared with a 31\% restoration of tensile strength in non-treated wounds. This corresponded with a more effective deposition of collagenous fibers that were also more highly organized. In short, scarring was reduced and there was a consequent increase in tissue function. The rabbits used in the study were fully immunocompetent, and no immunosuppressive drugs were given to the animals; there was no evidence for the rejection of injected xenogenic hMSCs.

Falanga and colleagues [19] approached the problem of skin wound repair differently. They incorporated autologous MSCs in a fibrin spray for topical delivery. This procedure concentrated the cells and provided a non-toxic matrix from which cells could migrate into wound beds and was applicable for use for both experimental animals and humans. In the experimental mouse model, excisional wounds were created on tail skin of genetically diabetic $(\mathrm{db} / \mathrm{db})$ mice, and fibrin spay was administered with and without autologous MSCs. The MSCs were tracked into the wound bed but most transited out of the wound by day 21 after application. A few isolated cells that remained in the wounds associated with vascular structures. Wounds treated with MSCs healed significantly faster and displayed a more mature histology than did wounds in which cells were not applied. Using autologous MSCs, the authors performed a similar study on human chronic non-healing wounds. A strong correlation was observed in the number of applied cells and the closure of the chronic wounds.

Nambu and colleagues [24] treated excisional wounds on $\mathrm{db} / \mathrm{db}$ mice with autologous ASCs that were incorporated in a collagen matrix and found advanced granulation tissue formation and re-epithelialization with applied cells as compared with controls. These results suggest that ASCs may share similar trophic effects with bone marrow-derived MSCs. Another potential method for the direct application of MSCs to skin wounds is through their incorporation into skin equivalents. Typical bilayered skin equivalents consist of an epidermis that resides on a fibroblast-based dermis [28]. Since MSCs are a fibroblastic population, they can be used either alone or in combination with dermal fibroblasts to construct the dermal component of skin equivalents [27]. Integration of MSCs into a fibroblast matrix has been shown to enhance the angiogenic potential of that matrix [29]. This implies that these cells might retain at least some of their MSC characteristics. Alternatively, MSCs might acquire a more fibroblast-like phenotype [9].

\section{How do mesenchymal stem cells improve wound healing?}

The basic mechanisms by which MSCs might improve wounds are (a) paracrine communication with resident wound cells, infiltrating inflammatory cells, and antigenpresenting cells or (b) their differentiation into resident cells or (c) both [30,31]. If paracrine activity is their primary function in wound repair, their presence in wounds would be expected to be transitory. However, if they differentiate into structural tissue cells such as fibroblasts, vascular endothelial cells, or pericytes, their presence would be expected to be longstanding. Current information on MSC longevity in wounds varies. For example, Falanga and colleagues [19] found that most of their topically applied MSCs had exited the wound by 25 days. In contrast, injection of MSCs into the pericardiac region revealed the continuous presence of MSCs for up to 1 year [32]. These variations may depend on the type of tissue, type of wound, the method of labeling cells, or the degree of MSC heterogeneity.

\section{Fate of mesenchymal stem cells delivered to wounds}

The long-term fate of therapeutic cells in wounds is not well characterized. Falanga and colleagues [19] reported that nearly all MSCs topically applied to mouse skin wounds exited the wounds prior to the completion of healing. In contrast, Yamaguichi and colleagues [33] found that topically applied MSCs differentiated to a myofibroblast phenotype in rat skin/fascia wounds. The differentiation of MSCs into fibroblasts is normally difficult to prove since these two types of cells share cell surface markers. However, a recent study from Mao and colleagues [9] showed that subsets of cloned MSCs are capable of acquiring fibroblast characteristics when treated with connective tissue growth factor. These cells do not express alpha-smooth muscle actin unless they are further treated with transforming growth factor-beta-1. In most studies, the long-term assessment of MSC fate is difficult without resorting to non-invasive techniques. Wang and colleagues [32] demonstrated that human CD34 ${ }^{+}$MSCs that were injected into a mouse myocardial infarct model were retained in the wound region for up to 52 weeks. The injected cells were labeled with luciferase vector, and a combination of bioluminescence, positron emission tomography, and magnetic resonance imaging was used to monitor cellular location. These cells 
did not appear to differentiate into myocardiocytes. Assessment of therapeutic cell fate will be important in future studies aimed at better understanding the function of these cells in wounds.

\section{Paracrine interactions}

Paracrine interactions that involve MSCs in a complex wound setting require these cells to be capable of producing and responding to a wide assortment of bioactive factors [34,35]. Furthermore, paracrine interactions imply close cellular juxtapositioning with communicating cells, a feature that would require integration of MSCs into the wound bed. A detailed understanding of the nature of these paracrine interactions will require combined in vivo and in vitro studies. Smith and colleagues [36] found that hMSCs in co-culture with dermal fibroblasts influenced the proliferation kinetics, migration kinetics, and gene expression profiles of these cells. However, wounds contain multiple subpopulations of fibroblasts that might interact differently than generic dermal fibroblasts [25]. Thus, the use of wound fibroblasts in such studies would be more informative. Studies have also shown that MSC therapy increases woundbreaking strength. This entails the production, deposition, and organization of structural collagens in wounds $[23,30]$. Therefore, it will be necessary to better understand how MSCs regulate collagen formation and metabolism in a fibroblast co-culture setting.

\section{Mesenchymal stem cell interactions with vasculature}

The interrelationship between MSCs and the vasculature is another area of relevance for wound repair. Granulation tissue formation is a critical early step in the healing process [25]. One of the therapeutic functions of MSCs is the early induction of granulation tissue [37-40]. This is followed by the stabilization of the neovascular network as wounds begin to heal. A current theory of MSC and ASC origin places these cells in perivascular domains in their respective organs [41-44]. In addition, analyses of newly isolated MSCs and ASCs have shown that these cells express markers characteristic of pericytes $[44,45]$. The native pericyte function of these cells may be retained in wound tissues. Pericytes are microvascular support cells that exhibit phenotypic characteristics intermediate between myofibroblasts and smooth muscle cells [40]. They partially envelop microvascular tubules and establish both $\mathrm{N}$-cadherin adherent junctions and communicating junctions with microvascular endothelial cells of tubules [46]. The neovasculature attracts pericytes through the release of the chemokines platelet-derived growth factor-BB (PDGF-BB). This interaction could explain, at least in part, the motive force behind MSC migration into wound tissue. Transforming growth factor-beta-1 plays a role in stabilization of pericyteendothelial cell complexes, and pericytes release angiopoitin-1, which helps to stabilize newly formed tubular structures through ligation with the Tie-2 receptor on endothelial cells $[47,48]$. Thus, therapeutic functions of MSCs in wounds likely include early induction of granulation tissue and stabilization of neovasculature.

Vascular stabilization by MSCs has been demonstrated in athymic mice in which mixtures of human umbilical vein endothelial cells (HUVECs) and hMSCs were implanted either in subcutaneous sites or into the cranial cavity $[45,49]$. As these cells sorted out, HUVECs formed vascular structures and MSCs assumed pericyte locations and expressed pericyte markers. The MSCs appeared to be attracted to the tubules by PDGF-BB [45]. Injection of endothelin-1 induced contraction of the human neovasculature, an indication of pericyte function. In longterm in vivo studies, vascular tubules retained their stability only when MSCs were present. In vitro coculture studies have also shown that MSCs increase tubule formation by HUVECs and that these MSCs assume pericyte-like positions on the tubules that these endothelial cells formed [29]. Taken together, these studies suggest that major therapeutic functions of MSCs and ASCs are to induce early granulation tissue formation and to stabilize neovasculature at wound sites.

\section{Mesenchymal stem cell modulation of immune and rejection responses}

MSC cellular interactions transcend the interactions with vascular endothelial cells, and the potent immunomodulatory activities of MSCs augment other repair functions through multifold, complex steps. The first step in MSC immunomodulation requires the activation of these cells with interferon-gamma, which is presented in combination with other factors such as tumor necrosis factoralpha or interleukin-1 [50]. This activation results in the upregulated release of soluble factors such as indoleamine 2,3-dioxygenase, interleukin-10, and prostaglandin $\mathrm{E}_{2}$. In proper combinations, factors released by stimulated MSCs suppress the mitogen-induced proliferation of both $\mathrm{CD}^{+}$and $\mathrm{CD}^{+} \mathrm{T}$ cells [51]. These factors may also promote the generation of immunoregulatory $\mathrm{T}$ cells and functionally interact with natural killer cells and immature dendritic cells [50]. These activities have therapeutic potentials, some of which have already been documented in preclinical and clinical studies $[2,5,7,52]$.

MSC/ASC introduction into wound/ischemic sites via the direct/topical approach also raises issues of whether these cells can exert similar immunomodulatory skills. For example, these cells may increase tolerance for the engraftment of skin equivalents constructed from allogeneic cells and help to promote vascular ingrowth 
into the graft $[7,50,52,53]$. They may also play a role in the reduction of scar formation. Dendritic cells and a specialized fibrocyte population that migrates to wounds appear to play roles in hypertrophic scar formation [54]. The presence of MSCs/ASCs may dampen the effects of these cells, thus reducing scarring. As reported above, Stoff and colleagues [23] found that xenogenic MSCs increased the tensile strength of healed incisional wounds. Apparently, this resulted from the more effective organization of newly produced collagenous fibers at the wound site. Thus, MSCs may also play a role in the regulation of matrix deposition and organization, which are important late-stage steps during wound repair [25]. In adults, wound repair is associated with atypical matrix deposition that may be related to the overproduction of factors such as transforming growth factor-beta-1 [55]. Modulation of growth factor expression at wound sites could regulate scar formation. Thus, MSCs may act at multiple stages of wound repair, but with different functions. The mechanism of delivery may not be critical in this regard.

The immunomodulatory functions of MSCs are important for clinical applications of these cells. First, these functions facilitate the application of allogeneic cells to wounds, thus creating the potential for off-theshelf products. Second, these functions may dampen overexuberant inflammatory responses that retard wound healing. Finally, they reduce scar formation through interactions with dendritic cells and fibrocytes that are associated with scar formation.

\section{Summary, conclusions, and clinical relevance}

A substantial body of current evidence supports the notion that MSCs and ASCs serve as therapeutic cells in wound/ischemic situations. Unfortunately, most of the existing studies are phenomenological. This means that there is still a considerable body of work required to address basic issues: What is the most effective means of delivering therapeutic cells to target sites? What are the cellular and molecular functions of these cells at their target sites? What is the ultimate fate of these cells upon the successful healing of the wound? Answers to these basic questions will engender improved approaches for cellular therapeutics. The timing of therapeutic cell delivery may be critical. Topical delivery provides a means to better regulate this aspect of their application. Cellular populations within wounds change depending on the phases of the repair process [25]. This means that therapeutic cells will encounter different microenvironments at each stage of the repair process. Thus, developing an understanding of both when and how to best deliver these cells to wound sites will be critical for maximizing their potential. Issues regarding the longterm safety of cellular therapy and whether off-the-shelf products can be effectively developed will also determine the future of this approach.

\section{Abbreviations}

ASC, adipose stromal cell/adipose stem cell; hMSC, human mesenchymal stem cell; HUVEC, human umbilical vein endothelial cell; MSC, mesenchymal stem cell; PDGF-BB, platelet-derived growth factor-BB.

\section{Competing interests}

The authors declare that they have no competing interests.

\section{Acknowledgments}

We gratefully acknowledge the financial support of the David and Virginia Baldwin Fund. This research was sponsored by the Armed Forces Institute of Regenerative Medicine (award W81XWH-08-2-0034). The US Army Medical Research Acquisition Activity (820 Chandler Street, Fort Detrick, MD 217025014 , USA) is the awarding and administering acquisition office. The content of this article does not necessarily reflect the position or policy of the

Government, and no official endorsement should be inferred.

Published: 24 September 2010

\section{References}

1. Caplan Al, Dennis JE: Mesenchymal stem cells as trophic mediators. J Cell Biochem 2006, 98:1076-1084.

2. Caplan Al: Why are MSCs therapeutic? New data: new insight. J Pathol 2009, 217:318-324.

3. Friedenstein AJ, Petrakova KV, Kurolesova Al, Frolova GP: Heterotopic of bone marrow: analysis of precursor cells for osteogenic and hematopoietic tissues. Transplantation 1968, 6:230-247.

4. Till JE, McCulloch EA: Hemopoietic stem cell differentiation. Biochim Biophys Acta 1980, 605:431-459.

5. Karp JM, Teo GSL: Mesenchymal stem cell homing: the devil is in the details. Cell Stem Cell 2009, 4:206-216.

6. Dash NR, Dash SN, Routray P, Mohapatra S, Mohapatra PC: Targeting nonhealing ulcers of lower extremity in human through autologous bone marrow-derived mesenchymal stem cells. Rejuvenation Res 2009, 12:359-368.

7. Zou Z, Zhang Y, Hao L, Wang F, Liu D, Su Y, Sun H: More insight into mesenchymal stem cells and their effects inside the body. Expert Opin Biol Ther 2010, 10:215-230.

8. Dominici M, Le Blanc K, Mueller I, Slaper-Cortenbach I, Marini F, Krause D, Deans R, Keating A, Prockop D, Horwitz E: Minimal criteria for defining mutipotent mesenchymal stromal cells. The International Society of Cellular Therapy position statement. Cytotherapy 2006, 8:315-317.

9. Lee CH, Shah B, Moioli EK, Mao JJ: CTGF directs fibroblast differentiation from human mesenchymal stem/stromal cells and defines connective tissue healing in a rodent injury model. J Clin Invest 2010, 120:3340-3349.

10. Guilak F, Lott KE, Awad HA, Cao Q, Hicok KC, Fermor B, Gimble JM: Clonal analysis of the differentiation potential of human adipose-derived adult stem cells. J Cell Physiol 2006, 206:229-237.

11. Traktuev DO, Merfeld-Clauss S, Li J, Kolonin M, Arap W, Pasqualini R, Johnstone BH, March KL: A population of multipotent CD34-positive adipose stromal cells share pericyte and mesenchymal surface markers, reside in a periendothelial location, and stabilize endothelial networks. Circ Res 2008, 102:77-85.

12. Zannetino ACW, Paton SAA, Khor F, Itescu S, Gimble JM: Multipotential human adipose-derived stromal stem cells exhibit a perivascular phenotype in vitro and in vivo. J Cell Physiol 2008, 214:413-421.

13. Hong SJ, Traktuev D, March KL: Therapeutic potential of adipose-derived stem cells in vascular growth and tissue repair. Curr Opinion Organ Transplant 2010, 15:86-91.

14. Crisan M, Yap S, Casteilla L, Chen C-W, Corselli M, Park TS, Andriolo G, Sun B, Zheng B, Zhang L, Norotte C, Teng P-N, Traas J, Schugar R, Deasy BM, Badylak S, Buhring H-J, Giacobino J-P, Lazzari L, Huard J, Péault B: A perivascular origin for mesenchymal stem cells in multiple human organs. Cell Stem Cell 2008, 3:301-313.

15. Corselli M, Chen C-W, Crisan M, Lazzari L, Péault B: Perivascular ancestors of adult multipotent stem cells. Aterioscler Thromb Vasc Biol 2010, 30:1104-1109.

16. Chamberlain G, Fox J, Ashton B, Middleton J: Concise review: mesenchymal 
stem cells: their phenotype, differentiation capacity, immunological features, and potential for homing. Stem Cells 2007, 25:2739-2749.

17. Mitchell JB, Mclntosh K, Zvonic S, Garrett S, Floyd ZE, Kloster A, Halvorsen YD, Storms RW, Goh B, Kilroy G, Wu X, Gimble JM: Immunophenotype of human adipose-derived cells: temporal changes in stromal-associated and stem cell-associated markers. Stem Cells 2006, 24:376-385.

18. Hamou C, Callaghan MJ, Thangarajah H, Chang E, Chang El, Grogan RH, Paterno J, Vial IN, Jazayeri L, Gurtner GC: Mesenchymal stem cells can participate in ischemic neovascularization. Plast Reconstr Surg 2009, 123:45S-55S.

19. Falanga V, Iwamoto S, Chartier M, Yufit T, Butmarc J, Kouttab N, Shrayer D, Carson P: Autologous bone marrow-derived cultured mesenchymal stem cells delivered in a fibrin spray accelerate healing in murine and human cutaneous wounds. Tissue Eng 2007, 13:1299-1312.

20. Hanson SE, Bentz ML, Hematti P: Mesenchymal stem cell therapy for nonhealing cutaneous wounds. Plast Reconstr Surg 2010, 125:510-516.

21. Badiavas EV, Abedi M, Butmarc J, Falanga V, Quesenberry P: Participation of bone marrow derived cells in cutaneous wound healing. J Cell Physiol 2003, 196:245-250.

22. Yoshikawa T, Mitsumo H, Nonaka I, Sen Y, Kawanishi K, Inada Y, Takakura Y, Okuchi K, Nonomura A: Wound therapy by marrow mesenchymal cell transplantation. Plast Reconstr Surg 2008, 121:860-877.

23. Stoff NS, Moore ST, Numnum M, Espinosa-de-los-Monteros A, Richter DF, Siegal GP, Chow LT, Feldman D, Vasconez LO, Mathis JM, Stoff A, Rivera A, Banerjee A, Stoff-Khalili MA, Curiel DT: Promotion of incisional wound repair by human mesenchymal stem cell transplantation. Exp Dermatol 2009, 18:362-369.

24. Nambu M, Kishimoto S, Nakamura S, Mizuno H, Yanagibayashi S, Yamamoto N, Azuma R, Nakamura S-I, Kiyosawa T, Ishihara M, Kanatani Y: Accelerated wound healing in healing-impaired $\mathrm{db} / \mathrm{db}$ mice by autologous adipose tissue-derived stromal cells combined with atelocollagen matrix. Ann Plast Surg 2009, 62:317-321.

25. Singer AJ, Clark RAF: Cutaneous wound healing. N Engl J Med 1999, 341:738-746.

26. Boyce ST, Warden MD: Principles and practices for treatment of cutaneous wounds with cultured skin substitutes. Am J Surg 2002, 183:445-456.

27. Fioretti F, Lebreton-DeCoster C, Gueniche F, Yousfi M, Humbert P, Godeau G, Senni K, Desmoulière A, Coulomb B: Human bone marrow-derived cells: an attractive source to populate dermal substitutes. Wound Repair Regen 2008, 16:87-94.

28. Boyce ST. Cultured skin substitutes: a review. Tissue Eng 1996, 2:255-266.

29. Sorrell JM, Baber MA, Caplan Al: Influence of adult mesenchymal stem cells on in vitro vascular formation. Tissue Eng Part A 2009, 15:1751-1761.

30. McFarlin K, Gao X, Liu Y, Dulchavsky DS, Li y, Chopp M, Dulchavsky S, Gautam SC: Bone marrow-derived mesenchymal stromal cells accelerate wound healing in the rats. Wound Repair Regen 2006, 14:471-478.

31. Wu Y, Chen L, Scott PG, Tredget EE: Mesenchymal stem cells enhance wound healing through differentiation and angiogenesis. Stem Cells 2007, 25:2648-2659.

32. Wang J, Zhang S, Rabinovich B, Bidant L, Soghomonyan S, Alauddin MM, Bankson JA, Shpall E, Willerson JT, Gelovani JG, Yeh TH: Human CD34+ cells in experimental myocardial infarction: long-term survival, sustained functional improvement, and mechanism of action. Circ Res 2010 106:1904-1911

33. Yamaguchi Y, Kubo T, Murakami T, Takahashi M, Hakamata Y, Kobayashi E, Yoshida S, Hosokawa K, Yoshikawa K, Itami S: Bone marrow cells differentiate into wound myofibroblasts and accelerate the healing of wounds with exposed bones when combined with an occlusive dressing. Br J Dermatol 2005, 152:616-622

34. da Silva Meirelles L, Fontes AM, Covas DT, Caplan Al: Mechanisms involved in the therapeutic properties of mesenchymal stem cells. Cytokine Growth Factor Rev 2009, 20:419-427.

35. Liu Y, Dulchavsky DS, Gao X, Kwon D, Chopp M, Dulchavsky S, Gautam SC: Wound repair by bone marrow stromal cells through growth factor production. J Surg Res 2006, 136:336-341.

36. Smith AN, Willis E, Chan VT, Muffley LA, Isik FF, Gibran NS, Mocking AM: Mesenchymal stem cells induce dermal fibroblast responses to injury. Exp Cell Res 2010, 316:48-54.
37. Tögel F, Weiss K, Yang Y, Hu Z, Zhan, P, Westenfelder C: Vasculotropic, paracrine actions of infused mesenchymal stem cells are important to the recovery from acute kidney injury. Am J Physiol 2007, 292:F1626-F1635.

38. Egaña JT, Fierro FA, Krüger $S$, Bornhäuser M, Huss R, Lavandero $S$, Machens H-G: Use of human mesenchymal cells to improve vascularization in a mouse model for scaffold-based dermal regeneration. Tissue Eng Part A 2009, 15:1191-1200.

39. Tille J-C, Pepper MS: Mesenchymal cells potentiate vascular endothelial growth factor-induced angiogenesis in vitro. Exp Cell Res 2002, 280:179-191.

40. Gruber R, Kandler B, Holzmann P, Vögele-Kadleetz M, Losert U, Fisher MB, Watzek G: Bone marrow stromal cells can provide a local environment that favors migration and formation of tubular structures of endothelial cells. Tissue Eng 2005, 11:896-903.

41. Bianco P, Riminucci M, Gronthos S, Robey PG: Bone marrow stromal stem cells: nature, biology, and potential applications. Stem Cells 2001, 19:180-192.

42. Lamagna C, Bergers $\mathrm{G}$ : The bone marrow constitutes a reservoir of pericyte progenitors. J Leukoc Biol 2006, 80:677-681.

43. Caplan Al: All MSCs are pericytes? Cell Stem Cell 2008, 3:229-230.

44. Traktuev DO, Prater DN, Merfeld-Clauss S, Saneevaiah AR, Saadatzadeh MR, Murphy M, Johnstone BH, Ingram DA, March KL: Robust functional vascular network formation in vivo by cooperation of adipose progenitor and endothelial cells. Circ Res 2009, 104:1410-1420.

45. Au P, Tam J, Fukumura D, Jain RK: Bone marrow-derived mesenchymal stem cells facilitate engineering of long-lasting functional vasculature. Blood 2008, 111:4551-4558.

46. Gerhardt $\mathrm{H}$, Betsholtz $\mathrm{C}$ : Endothelial-pericyte interactions in angiogenesis. Cell Tissue Res 2003, 314:15-23.

47. Jain RK, Booth MF: What brings pericytes to tumor vessels? J Clin Invest 2003, 112:1134-1136.

48. Hirschi KK, Rohusky SA, D'Amore PA: PDGF, TGF $\beta$, and heterotypic cell-cell interactions mediate endothelial cell-induced recruitment of $10 T 1 / 2$ cells and their differentiation to a smooth muscle fate. J Cell Bio/ 1998, 141:805-814.

49. Sanz L, Santos-Valle P, Alonso-Camino V, Salas C, Serrano A, Vicario JL, Cuesta AM, Compte M, Sánchez-Martín D, Alvarez-Vallina L: Long-term in vivo imaging of human angiogenesis: critical role of bone marrow-derived mesenchymal stem cells for the generation of durable blood vessels. Microvasc Res 2008, 75:308-314.

50. Ghannam S, Bouffi C, Djouad F, Jorgensen, C, Noël D: Immunosuppression by mesenchymal stem cells: mechanisms and clinical applications. Stem Cell Res Ther 2010, 1:2.

51. Le Blanc K, Tammik L, Sundberg B, Haynesworth SE, Ringden O: Mesenchymal stem cells inhibit and stimulate mixed lymphocyte cultures and mitogenic responses independently of the major histocompatibility complex. Scand J Immunol 2003, 57:11-20.

52. Beggs KJ, Lyubimov A, Borneman JN, Bartholomew A, Moseley A, Dodds R, Archambault MP, Smith AK, McIntosh KR: Immunologic consequences of multiple, high-dose administration of allogeneic mesenchymal stem cells to baboons. Cell Transplant 2006, 15:711-721.

53. Bartholomew A, Sturgeon C, Siatskas M, Ferrer K, McIntosh K, Patil S, Hardy W, Devine S, Ucker D, Deans R, Moseley A, Hoffman R: Mesenchymal stem cells suppress lymphocyte proliferation in vitro and prolong skin graft survival in vivo. Exp Hematol 2002, 30:42-48.

54. Yang L, Scott PG, Dodd C, Medina A, Jiao H, Shankowsky HA, Ghahary A, Tredget EE: Identification of fibrocytes in postburn hypertrophic scar. Wound Repair Regen 2005, 13:398-404.

55. Shah M, Foreman DM, Ferguson MWJ: Neutralisation of TGF- $\beta 1$ and TGF- $\beta 2$ or exogenous addition of TGF- $\beta 3$ to cutaneous rat wounds reduces scarring. J Cell Sci 1995, 108:985-1002.

doi:10.1186/scrt30

Cite this article as: Sorrell JM, Caplan Al: Topical delivery of mesenchymal stem cells and their function in wounds. Stem Cell Research \& Therapy 2010, $1: 30$ 